\title{
Nonreciprocal and magnetically scanned miniaturized leaky-wave antennas using coupled transmission lines
}

\author{
Nil Apaydin, Kubilay Sertel, and John L. Volakis \\ ElectroScience Laboratory, Dept. of Electrical and Computer Engineering, The Ohio State University, 1330 Kinnear Road, \\ Columbus $\mathrm{OH} 43212$, USA
}

Received 29 October 2013 / Accepted 21 April 2014

\begin{abstract}
This paper presents a new class of magnetically scanned leaky wave antennas (LWAs), incorporating ferrite (or possibly magnetoelectric composite), for wide angle beamsteering. Using the ferrite's tunable permeability beamsteering is achieved by controlling the external bias field. This is unlike most leaky-wave antennas requiring frequency modulation to steer the beam. Our first design is based on coupled microstrip lines on a biased ferrite substrate with nonreciprocal radiation properties, specifically a $5 \mathrm{~dB}$ contrast between the measured transmit and receive gain in the E-plane was achieved. However, it was found that inhomogeneities in the bias field limited its scanning performance. To alleviate this issue, a new class of miniaturized metamaterial based LWA was considered and presented here. This new design is based on coupled composite right left handed (CRLH) transmission lines (TLs) and has a unit-cell length of only $\lambda_{0} / 20$. For validation, a 15 -unit-cell prototype was manufactured and its TX/RX beams were scanned in the $E$-plane $80^{\circ}$ by changing the bias field within a range of \pm 50 Oe. We found that the associated antenna gain varied between $3.5 \mathrm{~dB}$ and $5 \mathrm{~dB}$ at $1.79 \mathrm{GHz}$ as the beam was scanned. In the above design, scanning was realized by changing the distance between the bias source and the LWA. Thus, future work will be focused on LWAs tuned by biasing a magnetodielectric layer placed below the ferrite substrate.
\end{abstract}

Key words: Leaky-wave antenna, Frequency independent scanning, Wide-angle beamscanning, Ferrite.

\section{Introduction}

The demand for small, light-weight and multi-functional wireless devices continues to increase, fuelled by growth in the commercial and defense application. In this context, use of novel materials has become a new frontier for smaller and multifunctional integrated RF devices. Indeed, material mixtures and metamaterials at microwave frequencies have become an extensive research area [1]. For example, ferrites [2], and ferroelectrics [3] were exploited for phase shifters [4], antenna miniaturization [5], and for beam-scanning.

In this paper, we present recent work on novel miniaturized leaky wave antennas (LWAs), comprised of a pair of coupled TLs on a low-loss ferrite substrate. An external bias field control $\left(H_{0}\right)$ is used to control radiation and wide angle beamscanning. Antenna beam steering is traditionally done using phased arrays [6] or LWAs that support fast-waves [7]. In the case of phased arrays, high cost and complexity are inevitable due to the needed phase shifters at each element. When using LWAs, most available designs employ frequency modulation to achieve beam steering [7], implying additional challenges for

*e-mail: apaydin.3@buckeyemail.osu.edu
RF sources. Electronic beamscanning has been previously realized using varactors within the LWAs, but these varactors introduce losses that deteriorate antenna performance as the beam is scanned [8]. Among those, Chang et al. [9] have used pin diodes as switches to control the guided-wavelength. In this case, the dual state of the pin diode necessitates two discrete radiation angles instead of continuous scanning. As can be realized, these designs incorporate discrete circuit elements, making fabrication and biasing a challenge. Also, they have lave losses, making their radiation performance poor.

Mechanically tuned leaky-wave antennas have been considered as well [10]. However, mechanical tuning is usually slow and power consuming. To alleviate these issues, ferrites have been incorporated in LWAs to realize continuous scanning by controlling the magnetic material properties with an external magnetic field, $H_{0}[11,12]$. Magnetic tuning offers wide-angle and continuous scanning without appreciable gain degradation. Several other authors have also considered ferrites for scanning and nonreciprocal radiation [13-17]. However, challenges still remain in increasing antenna efficiency, improving non-reciprocity, tuning radiation, and minimizing the antenna size and cost. 
In our first design, leaky-wave radiation is attained by exciting the lowest fast harmonic of the dominant slow-wave mode. Unlike guided waves, fast waves are loosely bound to the antenna and radiate power as they propagate along the LWA. An 8-unit-cell leaky-wave antenna (LWA) was constructed and tested in presence of an external magnetic field to demonstrate its nonreciprocal performance. However, due to the nonuniformity in the bias field, only a $5 \mathrm{~dB}$ contrast was obtained between the measured transmit and receive gain in the $E$-plane. Further, beamscanning couldn't be validated.

To reduce the effect of bias field nonuniformities, a new class of miniaturized LWA is proposed herewith based on CRLH TLs. The latter incorporates series capacitors and shunt inductors to support dominant fast waves at low frequencies and lead to significantly miniaturized LWAs [18]. This CRLH $\mathrm{TL}$ on a ferrite substrate does not employ the higher order harmonics of the fundamental slow-wave mode [7]. Instead, higher order dispersion relations due to mode coupling were exploited to increase the LWA's sensitivity to the external bias field $H_{0}$ to attain wide angle scanning using a small variation/range of the bias field.

To validate the beam-steering range of the proposed miniature LWA, a 15 unit-cell prototype was manufactured and tested. Indeed, excellent beam steering was achieved over an $80^{\circ}$ span by changing $H_{0}$ across $H_{0} \pm 50 \mathrm{Oe}$. Additionally, $10 \mathrm{~dB}$ isolation was measured between TX and RX ports, validating the nonreciprocal properties of the proposed LWA. In these measurements, scanning was realized by changing the distance between the permanent magnet and the LWA. This approach is not practical as mechanical scanning is usually slow and power consuming. Therefore, a new class of LWA using magnetoelectrics is proposed for wide angle beam-steering. Specifically, a voltage $V_{\mathrm{DC}}$ is applied across the magnetoelectic piezo layer to introduce a mechanical deformation (expansion of the piezo surface). This deformation changes the magnetic field inside the ferrite layer $\left(H_{i}\right)$ due to the magnetostrictive strain at the interface [19]. As such, the magnetic properties of the ferrite (controlled by $H_{i}$ ) can be tuned by varying $V_{\mathrm{DC}}$ in the piezo layer to achieve beam-steering. The incorporation of these novel materials in antenna designs will be game changing. However, challenges remain in operating using small values of $V_{\mathrm{DC}}$ to realize optimal antenna performances with wide-band and directive radiation across large angular sector using only a small variation in the applied DC voltage.

\section{Periodic leaky-wave antenna basics}

Leaky-wave antennas are a class of antennas fed from a single feed that excites a longitudinally propagating fast wave with a phase constant $\beta_{z}$ that is less than free space wavenumber $k_{0}$, such that $\beta_{z}<k_{0}$, where $k_{0}$ is the free space wanenumber [7]. LWAs are attractive as they are low-profile and produce very narrow beams depending on the antenna size. They can also achieve beamscanning the radiated beam using different mechanisms (e.g. frequency, electronically, magnetically scanned).

Radiation of the LWA is achieved as the propagating wave along the antenna (see Figure 1) radiate power [7]. The direction of the radiated beam depends on the real value of

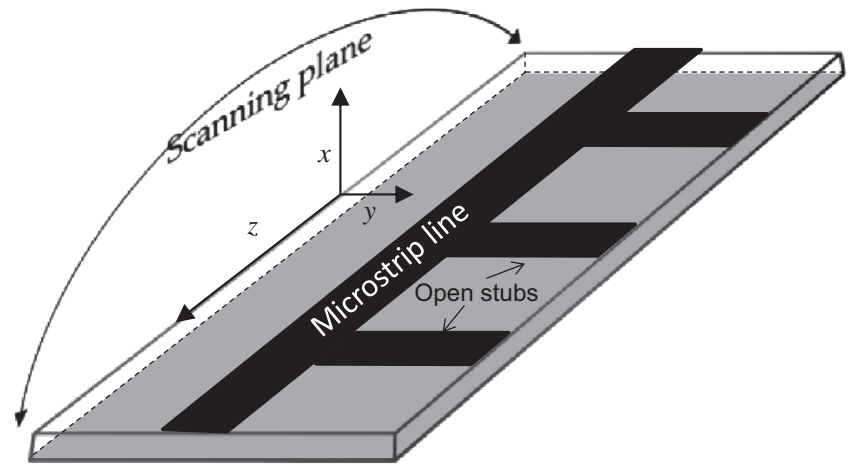

(a)

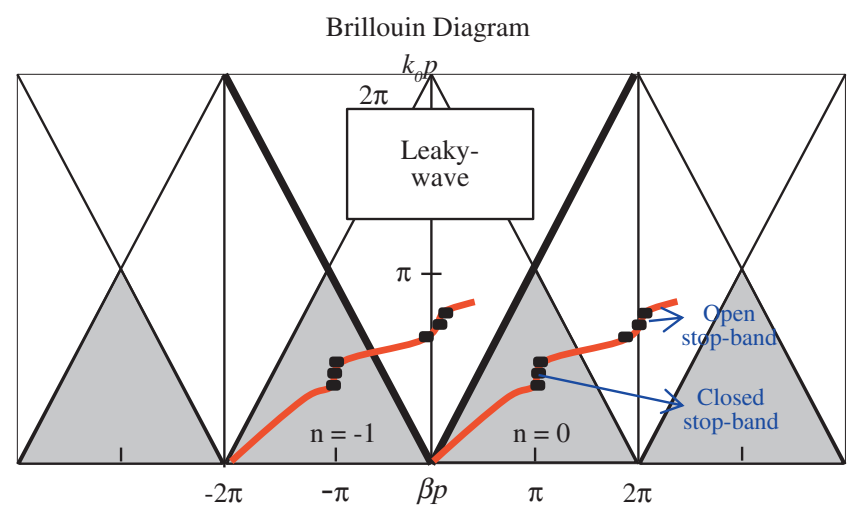

(b)

Figure 1. (a) Typical periodic LWA using a periodic arrangement of stubs along a microstrip line. (b) A representative brillouin $\left(\beta p-k_{0} p\right)$ diagram of a periodic leaky-wave antenna. Grey shaded region is the slow-wave region, where the modes are strongly bound to the surface and the region within the cone bounded by straight $\beta p= \pm k_{0} p$ lines, indicated by thick black solid lines, is the leakywave region, where radiation occurs.

the propagation constant $\beta_{z}$, whereas its radiation strength is dependent on the attenuation constant $\alpha_{z}$ viz. $k_{z}=\beta_{z}-j \alpha_{z}$ $k_{z}=\beta_{z}-j \alpha_{z}$. A large $\alpha_{z}$ implies that the incident power is leaked out quickly over a short length, resulting in small effective aperture and larger beamwidth [7]. On the contrary, small $\alpha_{z}$ implies long effective apertures, resulting in very narrow beams. It is important to note that the LWA's beam can be directed anywhere between the forward and backward endfire directions. Specifically, the beam radiation angle $\theta$ is given by

$$
\theta=\sin ^{-1} \beta_{z} / k_{0} .
$$

That is, the beam direction changes with frequency as $\beta_{z}$ is not necessarily a linear function of frequency.

In our designs considered here, leaky-wave antennas (e.g. Figure 1a) are mostly periodic slow-wave structures. The periodic structure generates and causes the first space harmonic of the dominant slow wave to be fast. This harmonic is associated with a phase constant

$$
\beta_{-1}=\beta_{0}-\frac{2 \pi}{d},
$$




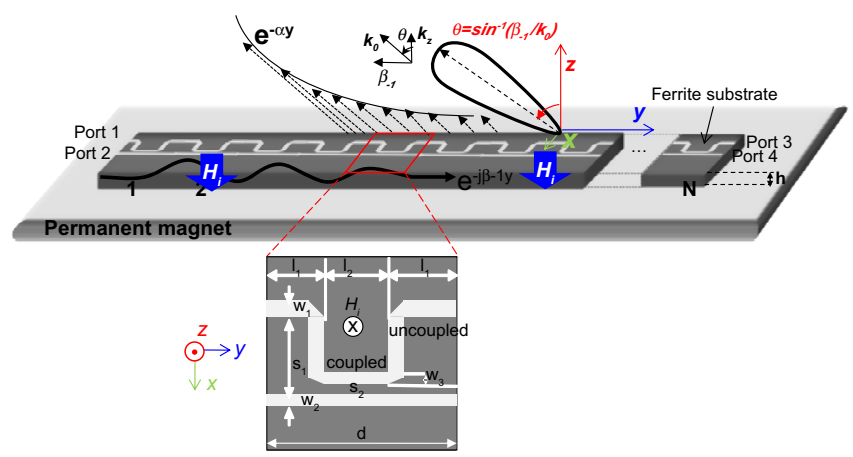

Figure 2. Coupled lines printed on a ferrite substrate having the material properties $4 \pi M_{s}=1000 \mathrm{G}$, loss linewidth $\Delta H=10 \mathrm{Oe}$, relative permittivity $\varepsilon_{r}=14$ and dielectric loss tangent $\tan \delta_{\varepsilon}=0.0002$. The internal DC magnetic field, $H_{i}$ is assumed to be in $z$ direction, normal to ground plane. Unit-cell dimensions are: $l_{1}=120, l_{2}=200$, $w_{1}=60, w_{2}=20, w_{3}=30, s_{1}=105, s_{2}=10$ (mils).

and radiates when

$$
-k_{0}<\beta_{-1}<k_{0}
$$

The beam can be scanned from backward to forward endfire by changing the frequency. As can be realized, to ensure that only a single beam is radiated over the operation band, $n=-2$ harmonic must not radiate. That is, it must remain a slow backward wave with $\beta_{-2}<-k_{0}$ with $\beta_{0}>k_{0}$.

To better understand the LWA's operation, we consider the dispersion diagram of the leaky wave line. Specifically, fast waves lie within the cone bounded by the straight line, $\beta p= \pm k_{0} p$, shown as thick black solid lines. When a space harmonic falls within this region, it will start radiating. We remark that the Brillouin diagram in Figure $1 \mathrm{~b}$ is comprised of an infinite number of identical regions repeated with a period of $2 \pi$ along the horizontal axis. Here, we only plot $n=0$ and $n=-1$ space harmonics with the latter simply being $-2 \pi$ shifted from the $n=0$ harmonic.

A typical issue with leaky-wave antennas is that an open stop-band occurs at $\beta_{-1}=0$ corresponding to $\beta_{0} p=2 \pi$, depicted in Figure 1b. The leakage constant $\alpha_{z}$ drops to 0 when $\beta_{0} p$ is exactly equal to $2 \pi$ and radiation is suppressed. For a practical finite sized antenna, this results in a significant drop in the LWA's gain when the beam is directed near broadside. Also most of the input power is reflected back to the source [20] due to mismatching. To address this issue, we used a ferrite substrate and shift the open stop-band region outside the leakywave region [11, 12]. Details of this design are given in next section.

\subsection{Nonreciprocal LWA based on coupled TLs on ferrite substrate}

Figure 2 shows an LWA design, comprised of a pair of coupled microstrip lines printed on a 100 mil thick commercially available CVG substrate. The latter has saturation magnetization $4 \pi M_{s}=1000 \mathrm{G}$, loss linewidth $\Delta H=10 \mathrm{Oe}$, relative permittivity $\varepsilon_{r}=14$ and dielectric loss tangent $\tan \delta_{\varepsilon}=0.0002$. We note that the narrow linewidth of the magnetic material was

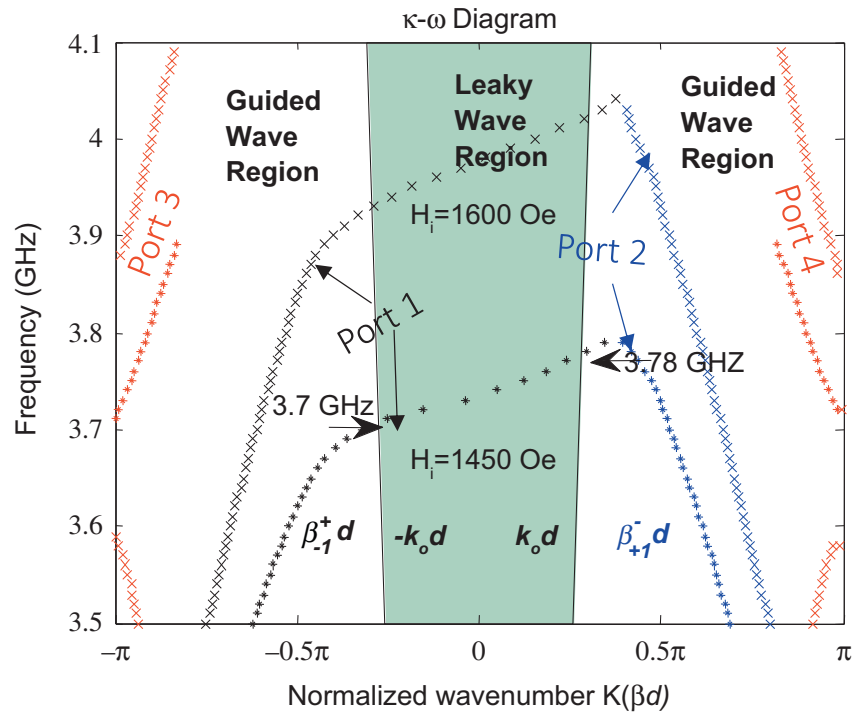

Figure 3. Dispersion diagram of the coupled microstrip lines unitcell in Figure 2. Calculations refer to $H_{i}=1450 \mathrm{Oe}$ (indicated with $*$ ) and 1600 Oe (indicated with $\times$ ).

specifically chosen to minimize losses. However, in practice, $\Delta H$ increases for non-uniformly biased substrates.

A unique feature of the proposed design is that the dispersion curves are spectrally asymmetric in the leaky-wave region. This was attained by to utilizing the field displacement effects with geometrical asymmetry of the unit-cell. Figure 3 depicts the $K-\omega$ dispersion curves obtained via the $T$-matrix method described in [21]. The red "*" and " $\times$ " marked curves in Figure 3 indicate refer to the modes supported on the straight transmission line. As seen, these modes are in the guided wave region and therefore do not radiate at the given frequency range. These modes also become leaky-wave modes at high frequencies but their leakage is very low as there is no line perturbation to cause radiation. Therefore, they are not efficient radiators. On the other hand, mitered bends on the meandered line introduce space harmonics of the fundamental mode at low frequencies and cause radiation.

The black and blue * and $\times$ marked curves in Figure 3 indicate the dispersion of $n=-1\left(\beta_{-1}^{+}\right)$and $n=+1\left(\beta_{+1}^{-}\right)$space harmonics. These refer to waves carrying power in $+z$ or $-z$ direction. Spectral asymmetry is clearly observed between $3.7 \mathrm{GHz}$ and $3.78 \mathrm{GHz}$ for $H_{i}=1450 \mathrm{Oe}$, with the $n=-1$ harmonic being the only fast wave. This leads to the proposed non-reciprocity in transmit and receive configurations. That is, a transmitting antenna, fed on the left side of the meandered line and terminated on the right side with a matched load, $Z_{L}=50 \Omega$, will start radiating a backward endfire beam at $3.7 \mathrm{GHz}$. As the frequency increases to $3.78 \mathrm{GHz}$. The LWA will then scan through the forward endfire direction. Meanwhile, spectral asymmetry mitigates the undesired backward radiation due to reflected waves $\left(\beta_{+1}^{-}>k_{0}\right)$. On the contrary, in the receive mode of the LWA, only forward waves will be supported on the meandered line $\left(\beta_{-1}^{+}<k_{0}\right)$. These will in turn be guided to the right side and dissipated at the matched load termination $Z_{L}$. However, there are still mismatches at the mitered bends and at the load, causing reflected waves. 
Therefore, some of the incident power is transmitted to the feed port. These mismatches need be minimized to attain maximum nonreciprocity.

Another important feature of the proposed design is that the open stop band starts when $\beta_{+1}^{-}=\beta_{-1}^{+}$is shifted to the guided wave regime. Therefore, input mismatches are avoided within the operation band.

For a finite LWA (that will be measured) to have low losses, the ferrite substrate must be fully saturated. To ensure this, the final LWA design needs to be shorter than the $6^{\prime \prime}$ long permanent magnet block used for biasing. Another consideration is that a finite LWA is usually designed such that $90 \%$ of the power is radiated, and this is in accordance with the formula:

$$
\frac{L}{\lambda_{0}}=\frac{0.18}{\frac{\alpha}{k_{0}}} .
$$

From (4), to attain $90 \%$ efficiency, $L$ needs to be larger than 1.8 $\lambda_{0}$ since $\alpha_{l} / k_{0}<0.1$ for this specific design. However, coupled power to the straight TL and material losses don't allow for $90 \%$ efficiency even though $L$ is chosen to be larger than 1.8 $\lambda_{0}$. In this work, $L$ will be $4^{\prime \prime}\left(1.34 \lambda_{0}\right)$, corresponding to an efficiency of $\sim 75 \%$. However, as mentioned before, bias field nonuniformities widen the magnetic loss line-width, $\Delta H$, and drop the efficiency to $50 \%$.

For the experimental validation of the above calculations, we manufactured an 8 unit-cell prototype on a $3.96^{\prime \prime} \times 0.44^{\prime \prime} \times 0.10^{\prime \prime}$ CVG substrate (obtained from TCI ceramics). The transmission lines were printed on the ferrite substrate using the AMI MSP-485 precision screen printer. Finally, the magnetic field was applied by the permanent magnet placed under the antenna, as depicted in Figure 2. The LWA was fed at port 1 , with the other unused ports terminated at $50 \Omega$ prior to measuring the radiation patterns.

Figures $4 \mathrm{a}-4 \mathrm{~d}$ show the measured TX and RX antenna patterns at $3.975 \mathrm{GHz}$ and $3.775 \mathrm{GHz}$. The first refers to the frequency where the maximum TX gain occurs and the other refers to the frequency where the maximum contrast between TX and RX gains appears. As seen, good agreement is observed between measurements and simulation. At $3.975 \mathrm{GHz}$, the transmitting beam is directed at $\theta_{M}=30^{\circ}$ in the backward direction with a half power beamwidth of $\Delta \theta=75^{\circ}$. The relatively large beamwidth is attributed to the variations in $H_{i}$ along the length of the antenna. At $3.775 \mathrm{GHz}$, the beam breaks into a pair of lobes, attributed to the asymmetrical external field distribution that causes radiation in the opposite direction at low frequencies. A maximum of $5 \mathrm{~dB}$ contrast is observed at $3.775 \mathrm{GHz}$ between the TX and RX gains. The transmitting antenna has a $-3 \mathrm{~dB}$ gain bandwidth of $13 \%$. We also observe that the TX antenna gain drops above $4 \mathrm{GHz}$ due to the ferromagnetic resonance of the ferrite substrate. Notably, the TX antenna radiates in LHCP, with a polarization ratio of $8 \mathrm{~dB}$ over the bandwidth. Simulated and measured data agree well at the high frequency end, but diverge as the frequency decreases likely due to the interference from the termination of the finite LWA. Of importance is that the measured curves follow the character of the computed ones. Additionally, the LWA has impedance bandwidth $\left(S_{11}<-10 \mathrm{~dB}\right)$ of $16 \%$.

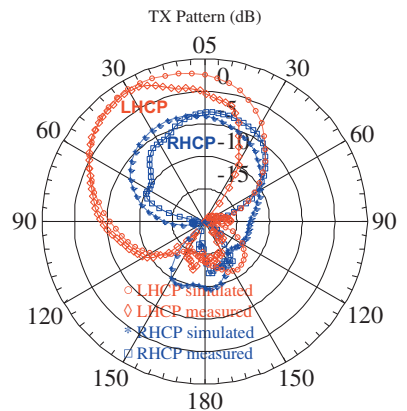

(a)

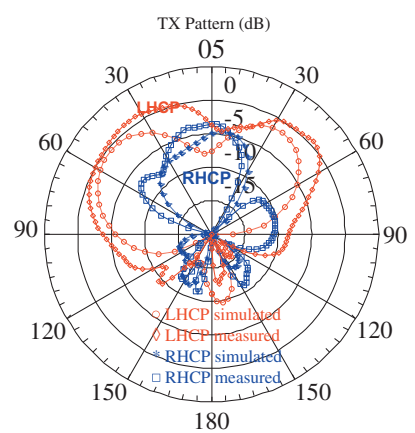

(c)

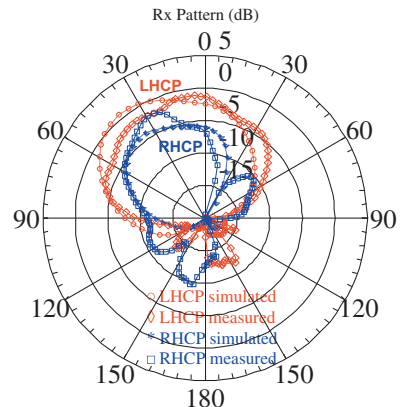

(b)

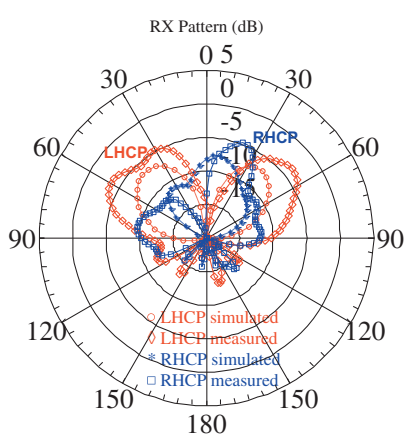

(d)
Figure 4. (a) Measured and simulated transmitting and (b) receiving antenna patterns (in the $y-z$ plane) at $3.975 \mathrm{GHz}$, (c) Measured and simulated transmitting and $(\mathrm{d})$ receiving antenna patterns (in the $y-z$ plane) at $3.775 \mathrm{GHz}$.

Although the above leaky-wave antenna has unique features such as nonreciprocal radiation, it had its downsides. For instance, the unit-cell was electrically long because the structure radiates only at higher frequencies through space harmonics. Similarly, the overall length of the antenna was limited by the size of available permanent magnets, restricting the number of unit-cells in the final leaky-wave antenna design and therefore deteriorating antenna performance. Another issue was the strong nonuniformity in the bias field, inhibiting the tunability of the ferrite substrate.

To reduce the effect of bias field nonuniformities on radiation performance, a new class of miniaturized nonreciprocal leaky-wave antennas based on coupled Composite Right Left Handed (CRLH) transmission lines (TLs) are introduced next. A unique feature of CRLH TLs is that they support dominant fast waves even at low frequencies. This property has long been utilized to design smaller leaky-wave antennas with very directive beams. When this concept is incorporated with coupled transmission lines, the resultant antenna provides even additional miniaturization, wide-angle scanning with minimal DC bias field control and different transmit and receive ports. The design details for this CRLH LWA are given next.

\section{Miniaturized and magnetically scanned leaky-wave antenna using coupled CRLH lines}

Previously [11, 12], the leaky-wave radiation was achieved by exciting the first fast space harmonic (associated with phase 

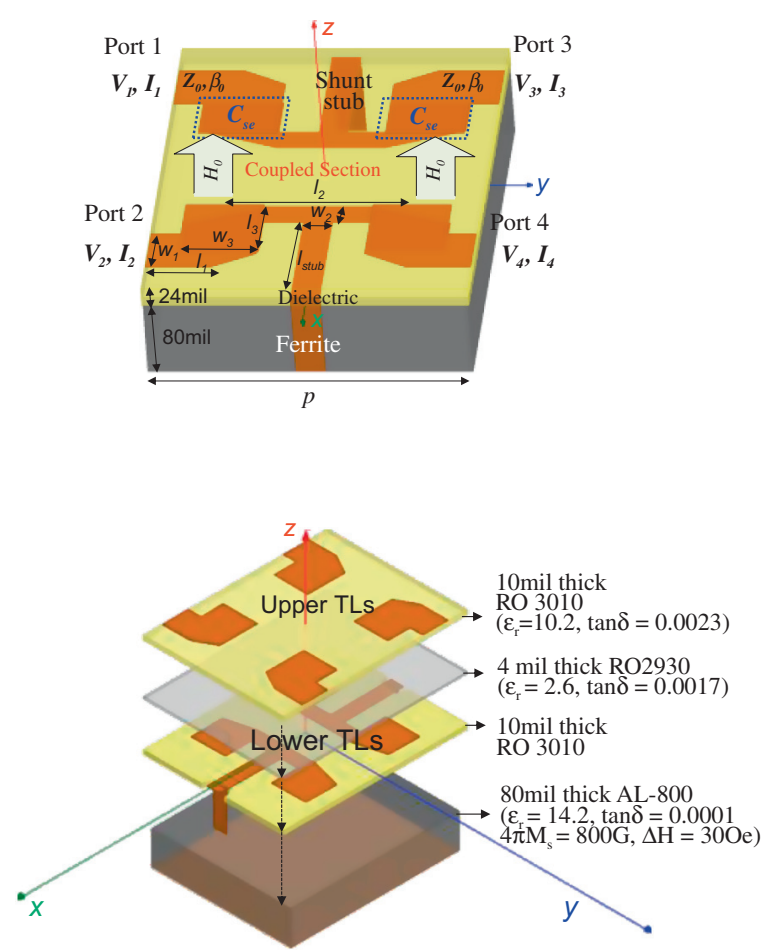

(a)

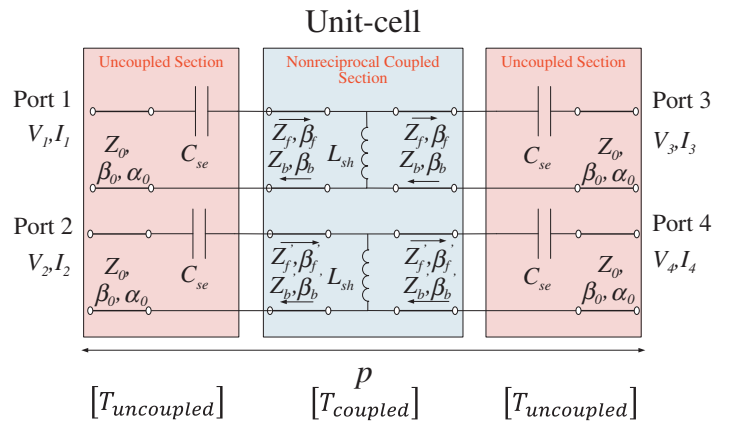

(b)

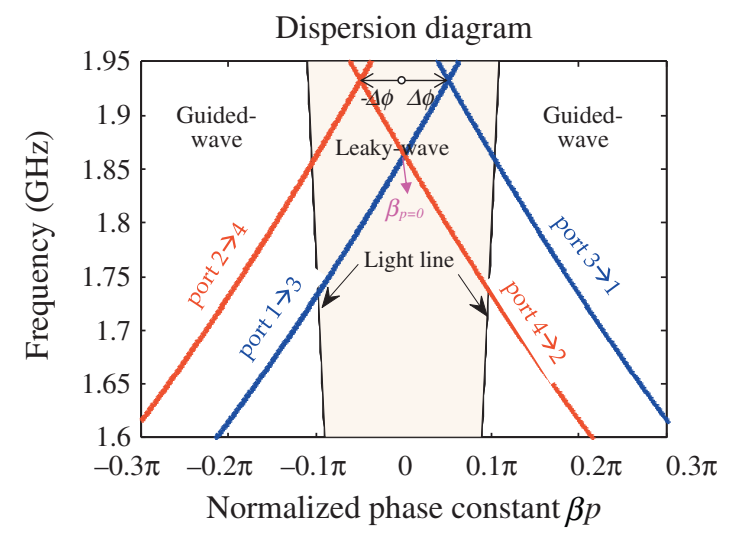

(c)

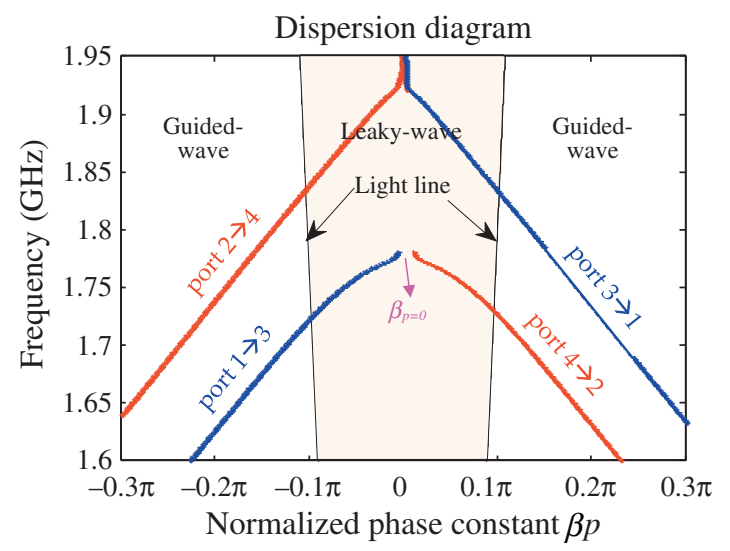

(d)

Figure 5. (a) Modified unit-cell geometry with series chip capacitors and shunt inductors to introduce low frequency resonance in the leakywave region of the $K$ - $\omega$ diagram. This approach allowed us to miniaturize the LWA design and to operate at $L$-band. Dimensions are: $l_{1}=105$, $l_{2}=120, w_{1}=60, w_{2}=30, w_{3}=80, l_{3}=75, l_{\text {stub }}=131.5$ (mils), and (b) its equivalent circuit model as a cascade of uncoupled and coupled sections to analytically calculate the unit-cell $T$-matrix. (c) Dispersion curves when coupling between the lines $\left(L_{m}=10^{-3} \mathrm{pH}, C_{m}=10^{-3} \mathrm{pF}\right)$ is negligible and (d) when $L_{m}=5.45 \mathrm{nH}, C_{m}=1.15 \mathrm{pF}$.

constant $\beta_{-1}=\beta_{0}-2 \pi / p$, where $\beta_{0}$ is the phase constant of dominant slow-wave and $p$ is the period) of the dominant slow-wave mode. This harmonic becomes fast and radiates only at high frequencies, causing the antenna to be electrically longer (several $\lambda_{0}$ long). Specifically, the LWA design in previous section was $1.34 \lambda_{0}$ long and had an efficiency of $50 \%$ [12]. To improve efficiency, we must increase the number of unit-cells in the LWA. However, as pointed out earlier, our LWA length is limited by the length of the available permanent magnet, needed to provide uniform magnetic field. A way to increase $\eta$ (without increasing the antenna length) is to miniaturize the unit-cell size, while retaining the same power leakage per unit-cell. As such, more unit-cells can be included in the LWA design, resulting in increased gain and efficiency. To this end, we considered inserting series chip capacitors and shunt stubs within the coupled TLs to introduce a low-frequency resonance in the fast-wave region of the $K$ - $\omega$ diagram (the concept is illustrated in Figure 5). These transmission lines are called Composite Right Left Handed (CRLH) transmission lines (TLs) and were extensively utilized to develop miniaturized 
LWA designs. Here, CRLH is integrated with the coupled-line concept to achieve further miniaturization and nonreciprocal radiation at the same time.

The unit-cell of the proposed design is depicted in Figure 5a, where a 24 mil thick PCB (with Rogers RT/Duroid 3010, $\left.\varepsilon_{r}=10.2, \tan \delta=0.0023\right)$ is used as a superstrate and placed on an 80 mil thick AL-800 ferrite. The latter substrate has saturation magnetization $4 \pi M \mathrm{~s}=800 \mathrm{G}$, loss linewidth $\Delta H=30 \mathrm{Oe}$, permittivity $\varepsilon_{r}=14.2$, dielectric loss tangent $\tan \delta=0.001$. An external DC magnetic field of strength $H_{0}$ is applied normal to the ground plane to control the ferrite's material properties and realize beamsteering without a need for frequency modulation [24].

The series capacitors $\left(C_{\mathrm{se}}\right)$ were realized using overlapping TL sections, giving us the advantage of attaining much larger capacitances and additional miniaturization as compared to designs with inter-digital capacitors. For instance, the total series capacitance $\left(2 C_{\mathrm{se}}\right)$ in each unit-cell was near $2.6 \mathrm{pF}$, difficult to realize using inter-digital capacitors.

When each TL is considered independently, the shunt stub introduces geometrical asymmetry, causing a phase shift between the forward and backward propagating waves $\left(\mathrm{e}^{-j \Delta \beta y}\right)$. This is because the fields are asymmetrically displaced to the left side of the TL for propagation in the $+y$ direction and to the right side for the opposite propagation direction due to nonreciprocal field displacement effects in the ferrite substrate. In [22], it was noted that this nonreciprocity can be accounted in the circuit model by assigning different phase constants $\beta_{f}$ ( $\beta_{f}^{\prime}$ for lower TL), $\beta_{b}\left(\beta_{b}^{\prime}\right)$ and characteristic impedances $Z_{f}$ $\left(Z_{f}^{\prime}\right), Z_{b}\left(Z_{b}^{\prime}\right)$ to forward and backward propagating waves. For our case, the shunt stubs placed on the opposite sides of the coupled transmission lines also cause a phase shift $\Delta \beta=\beta_{b}{ }^{\prime}-\beta_{f}^{\prime}$ for the lower TL. The same but negative phase shift is associated with the upper TL.

Figure $5 \mathrm{c}$ depicts the calculated dispersion curves for the unit-cell in Figure 5a assuming negligible coupling between the transmission lines. For this calculation, the bias field $H_{0}$ was assumed to be $80 \mathrm{kA} / \mathrm{m}(\sim 1000 \mathrm{Oe})$. The blue lines in the graphs of Figure 5 indicate the $\beta$ of the supported modes in the upper TL, whereas the red lines are for the lower TL. It is clearly observed that red and blue curves are identical but are displaced from $\beta p=0$ by $-\Delta \varphi$ and $\Delta \varphi$, respectively. This is because shunt stubs are located on the opposite sides of the corresponding TLs.

When these two TLs couple to each other, higher order dispersion relations are realized (i.e. 2 nd order near $\beta p=0$ ). Also, the sensitivity of $\beta$ to magnetic bias field $H_{0}$ improves. This is necessary to scan the beam using only a small amount of bias field change unlike other magnetically scanned antennas 13, 16. Figure $5 \mathrm{~d}$ depicts the simulated dispersion curves of the coupled CRLH unit-cell. It is noted that coupling between the lines push the dispersion curves (see $\beta p=0$ point) down to lower frequencies and lead to additional miniaturization. To calculate the effect of increased capacitance $\left(C_{\mathrm{se}}=2.6 \mathrm{pF}\right)$ and mode coupling on the miniaturization, the dispersion diagram of regular TLs was also calculated using a circuit model and compared to those of single and coupled CRLH TLs. It was found that the CRLH TLs reduced the $\beta p=0$ point from

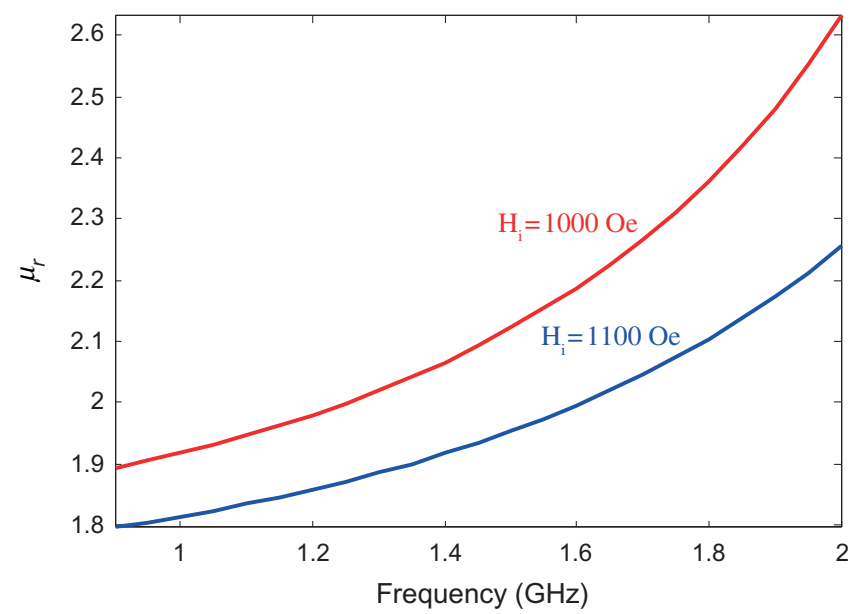

Figure 6. Relative permeability of AL- 800 with saturation magnetization $4 \pi \mathrm{Ms}=800 \mathrm{G}$, loss linewidth $\Delta H=30$ Oe calculated using Polder's tensor for internal magnetic field $H_{i}=1000$ and 1100 Oe.

$6.2 \mathrm{GHz}$ (for a regular TL) down to $1.85 \mathrm{GHz}$ (and $1.77 \mathrm{GHz}$ for the coupled TLs). This implies a miniaturization by a factor of 3.5. Additionally, the proposed unit-cell ( $\lambda_{0} / 20$ in length) is 2.65 times smaller than the unit-cell $\left(\lambda_{0} / 7.5\right.$ in length) of a regular CRLH LWA on a dielectric substrate [23].

Another important feature of the proposed antenna is its beamscanning capability by changing the external bias field, $H_{0}$. A key point in realizing wide angle beam-steering via small changes in $H_{0}$ is to operate the LWA relatively close to the ferromagnetic resonance (FMR) of the ferrite. FMR resonance is related to the internal magnetic field strength, $H_{i}$ as

$$
f_{\mathrm{FMR}}=\gamma H_{i},
$$

where $\gamma$ is $2.8 \mathrm{GHz} / \mathrm{kOe}$. For planar ferrite substrates [23]

$$
H_{i} \approx H_{0}-4 \pi M s .
$$

And ferrite's material properties are a function of $H_{i}, \beta$ of the fast waves can be controlled by changing the value of the external bias field $H_{0}$. When operating at a frequency is close to the FMR, $\mu_{r}$ of the ferrite becomes more sensitive to changes in $H_{0}$ and the scanning rate $\left(\Delta \theta_{m} / \Delta H_{0}\right)$ increase. As would be expected, when $\mu_{r}$ becomes $\sim 1$ and the LWA becomes insensitive to $H_{0}$. For this particular design, $H_{0}$ was chosen to be $\sim 1800 \mathrm{Oe}$, corresponding to $H_{i}\left(\approx H_{0}-4 \pi M s\right)$ of $1000 \mathrm{Oe}$. As a result, the FMR occurred at $2.8 \mathrm{GHz}$, whereas the operating frequency was $\sim 1.8 \mathrm{GHz}$. Higher sensitivity could be easily realized by bringing these two frequencies closer to each other. However, the loss linewidth of the AL-800 substrate was relatively large $(\Delta H=30 \mathrm{Oe})$, and therefore we preferred to operate away from FMR to avoid large magnetic losses. Permeability of the ferrite for $H_{i}=1000$ Oe and $H_{i}=1100 \mathrm{Oe}$ are depicted in Figure 6 as a function of frequency. For these $H_{i}$ values, the corresponding ferromagnetic resonances occur at $2.8 \mathrm{GHz}$ and $3.08 \mathrm{GHz}$, respectively. We note that the magnetic loss tangent was calculated to be $\sim 0.01$ at $1.8 \mathrm{GHz}$. As desired, the magnetic loss tangent is reasonably low. Also, the ferrite's relative permeability can be tuned $12 \% \quad\left(\mu_{r}=2.363\right.$ for $H_{i}=1000$ Oe and $\mu_{r}=2.1$ for $1100 \mathrm{Oe}$ ) by changing bias field 


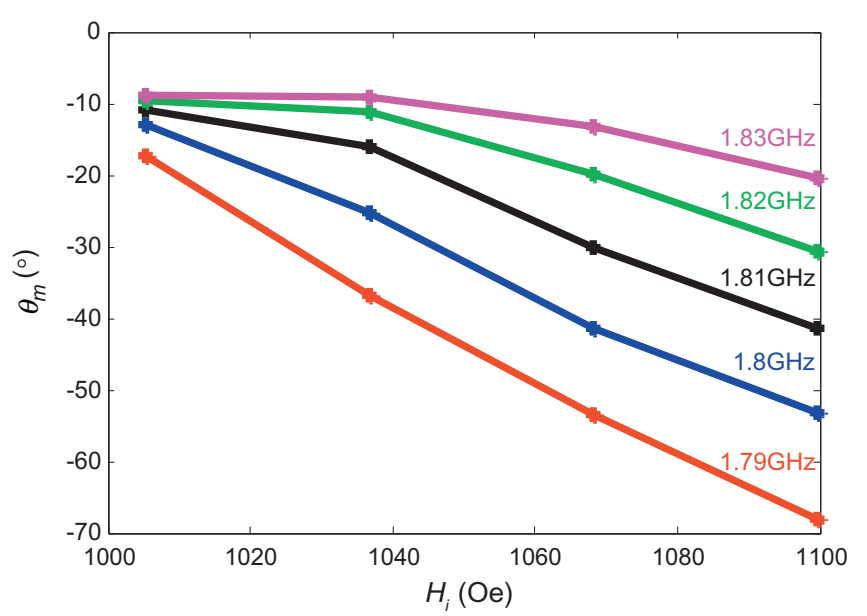

Figure 7. Scan angle $\theta_{m}$ plotted versus the bias field $H_{i}$ for different frequencies. Calculations were done using (2) and the dispersion curves were extracted via full-wave simulations.

a corresponding percentage of $10 \%$. We remark that tunability can be increased by operating closer to the FMR; however, the magnetic losses would also increase. Therefore, instead, we utilized the coupled CRLH TLs to increase the tunability range by realizing higher order dispersion relations (2nd, 3rd order).

The beam of the LWA can also scanned by varying the operating frequency. This is inevitable due to changes in the phase constant of the leaky wave mode as the frequency is varied. However, this imposes additional challenges for RF sources and large bandwidth designs. Therefore, it is important to control the bias field strength to cause scanning at a specific angle independent of frequency. To do so, we begin by examining the relationship between scan angle, frequency \& the bias field strength. As already noted, to retain the beam directed at a desired scan angle even as frequency changes, $\beta / k_{0}$ needs to be independent of frequency. Using our LWA, this can be done by modifying to be a function of frequency to counteract steering due to frequency. To do that, we must first find an analytical representation of $\beta$ as a function of the frequency $f$ and the bias field $H_{i}\left(\beta\left(H_{i} f\right)\right)$. The external bias field can then be changed accordingly.

It turns out that $\beta$ is not only dependent on $f$ and $H_{i}$ but is also strongly dependent on circuit parameters. Therefore, instead, we extracted the scan angle using the computed dispersion curves and plotted them versus the bias field strength at different frequencies. This is depicted in Figure 7, which can be used as a look-up plot to change the bias field as a function of frequency to maintain the beam direction. It is clearly observed from Figure 7 that scanning rate $\left(\Delta \theta_{m} / \Delta H_{i}=\right.$ $0.55^{\circ} / \mathrm{Oe}$ ) is larger at lower frequencies, whereas the scan angle doesn't change at all at higher frequencies. These should be kept in mind during measurements.

For the experimental validation, a 15 unit-cell LWA prototype was fabricated. To this end, a PCB was ordered from Multilayer Prototypes, comprised of a 10 mil thick Rogers RT/ Duroid 3010 dielectric layer with double sided printing. This layer was bonded to another 10 mil thick Rogers RT/Duroid 3010 dielectric layer using a 4 mil thick bond-ply with $\varepsilon_{r}=2.6$
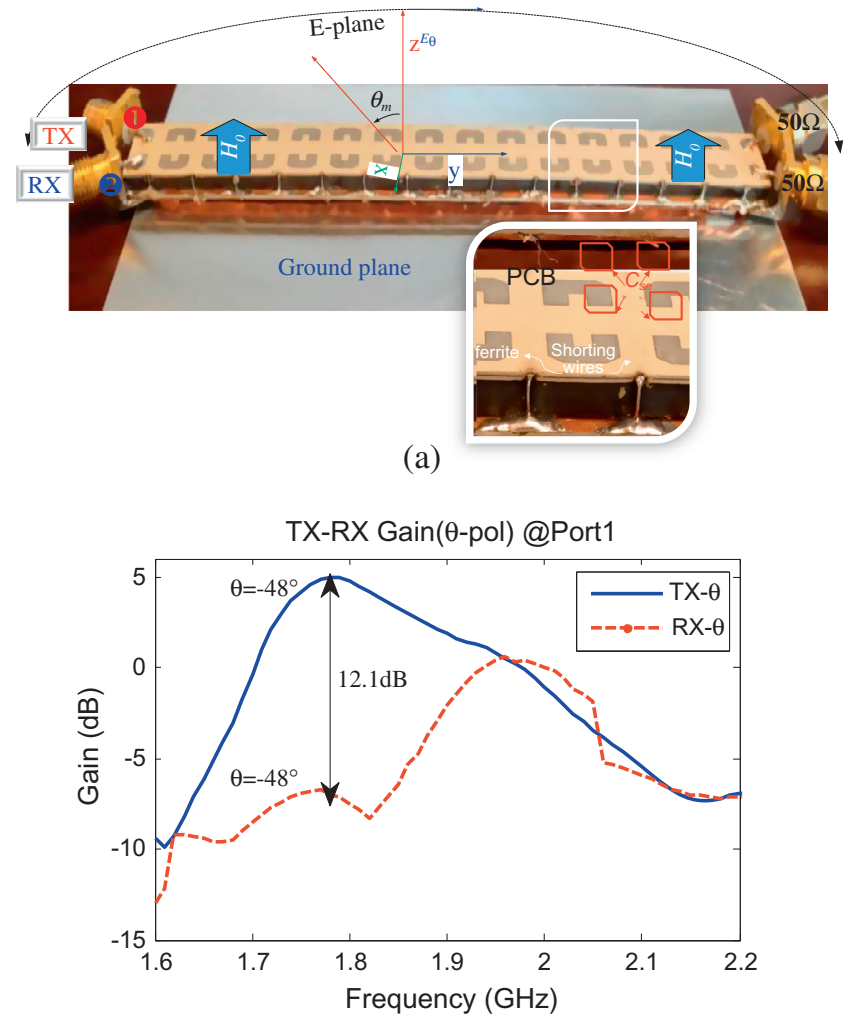

(b)

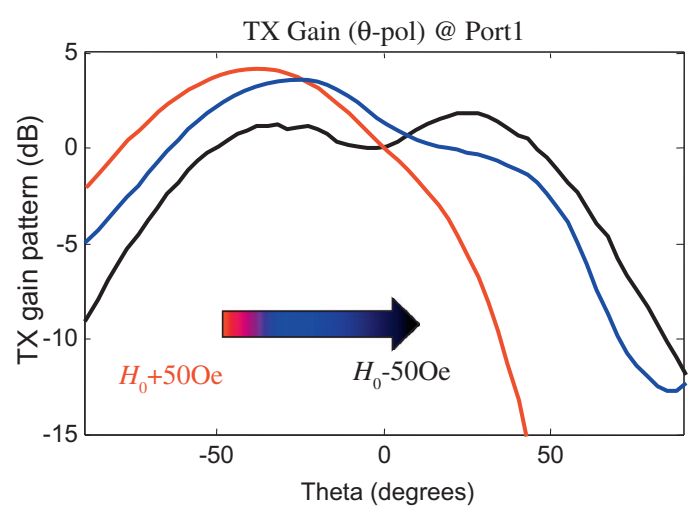

(c)

Figure 8. (a) Photo of the fabricated LWA prototype biased with an external magnetic field of $H_{0}=1900 \pm 150$ Oe. (b) Measured TX and RX gain patterns at port 1 and as a function of frequency, with port $3 \& 4$ terminated at $50 \Omega$. (c) Scanned TX antenna pattern at port 1 by changing the external bias field $H_{0}$ by \pm 50 Oe.

and $\tan \delta=10^{-3}$. This PCB was then placed on a $0.4^{\prime \prime} \times 4.95^{\prime \prime} \times 0.08^{\prime \prime}$ AL-800 ferrite substrate purchased from TCI ceramics. Stubs were shorted to the ground plane using AWG 26 copper wires.

Figure 8a depicts the final LWA, comprised of 15 unit-cells and $\sim \lambda_{0} / 70$ thick. This LWA was slightly shorter than the length of the permanent magnet and, thus, ensured that the ferrite was fully biased. For the experimental validation, the LWA prototype was tested in presence of $H_{0}$ supplied by a permanent magnet block $6^{\prime \prime} \times 3^{\prime \prime} \times 1.25^{\prime \prime}$ in size. The generated bias field by this magnet was $\sim 1900$ Oe with \pm 150 Oe variations in the 
longitudinal direction. Simulations showed that these variations didn't change the results significantly.

The TX and RX antenna patterns of the prototype were measured in the $E$-plane using port 1 and port 2 and are depicted in Figure 8b. A maximum of $12.1 \mathrm{~dB}$ contrast was observed between TX and RX antenna gains measured at port 1 . We note that port 1 was the TX port and port 2 was the RX port. The isolation between $\mathrm{TX}$ and $\mathrm{RX}$ ports was also measured to be more than $8 \mathrm{~dB}$ in the $1.65-1.8 \mathrm{GHz}$ frequency range.

The TX and RX antenna pattern was also scanned by controlling the bias field, $H_{0}$. The bias was changed by moving the magnet block at a variable distance from the LWA. Doing so, $H_{0}$ was varied \pm 50 Oe. The corresponding TX and RX patterns measured at port 1 are depicted in Figure $8 \mathrm{c}$ for $f=1.78 \mathrm{GHz}$. It is observed that the beam can be scanned from $\theta_{m}=-48^{\circ}$ to $35^{\circ}$ by simply decreasing the external bias field 100 Oe. During scanning, the antenna gain varied between $3.5 \mathrm{~dB}$ and $5 \mathrm{~dB}$ as the beam is scanned. We note that the beam splits at the lower bias fields because the antenna operates in the stop band region, where most of the power is coupled to the adjacent TL and radiated through modes propagating on this line. Summarizing, this innovative concept offers beam-steering at a single frequency and over a wide range of steering angles.

From the simulated data, we find that antenna efficiency is about $50 \%$ at $1.78 \mathrm{GHz}$ and drops at higher frequencies due to mode coupling. We remark that the scanning performance and efficiency of this antenna is still significantly better than other ferrite based antennas [14, 22], even though it is electrically short. The 4-port $S$-parameters were also measured using port 1 on the upper TL with the other 3 unused ports terminated at $Z_{L}=50 \Omega$. We note that $S_{11}<-10 \mathrm{~dB}$ between $1.7 \mathrm{GHz}$ and $1.8 \mathrm{GHz}$. Above $1.8 \mathrm{GHz}$, most of the power is coupled to port 2. This is because of the stop-band depicted in Figure 5c, which starts at $1.8 \mathrm{GHz}$. Minimum coupling, i.e. $S_{41}=$ $S_{21}=-8 \mathrm{~dB}$, to the lower TL occurs around $1.78 \mathrm{GHz}$.

As already noted, in this work, the beam was scanned by physically moving the permanent magnet away from the LWA to control/modify the bias field [24]. Clearly, this approach is not practical. Instead, electrical tuning is preferred. In next section, a new class of magnetoelectrics-based leakywave antennas (LWA) is proposed for wide angle beam-steering via electrical tuning.

\section{Electronically scanned leaky-wave antenna using magnetoelectric composite}

The proposed LWA is depicted in Figure 9 and is formed by a pair of coupled transmission lines printed on a magnetoelectric (ME) composite film, where ferrite and piezoelectric ceramic layers are mechanically coupled. That is, the voltage $V_{\mathrm{DC}}$ applied to the piezo layer introduces a mechanical deformation (expansion of the piezo surface). In turn, this deformation changes the magnetic field inside the ferrite layer $\left(H_{0}\right)$ due to the magnetostrictive strain at the interface $[19,25]$. As such, the magnetic properties of the ferrite (controlled by $H_{0}$ ) can be varied by changing the applied $V_{\mathrm{DC}}$ in the piezolayer to achieve beam-steering.

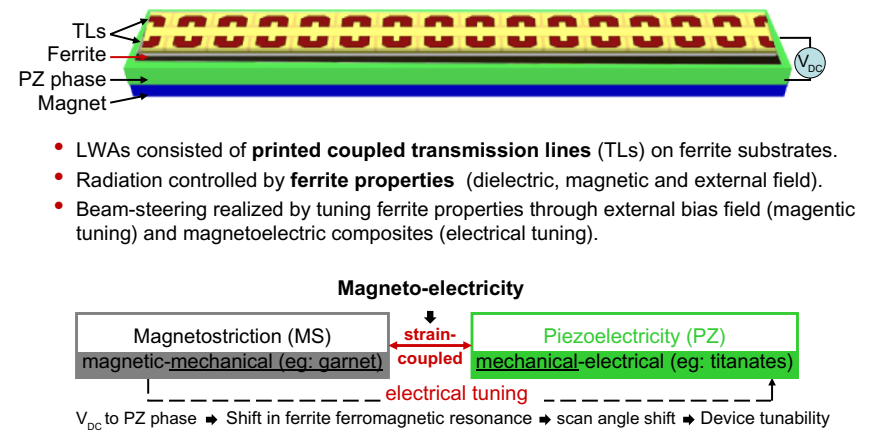

Figure 9. Layout of the magnetoelectic LWA comprised of ferrite, piezoelectric phase and magnets for electronic scanning.

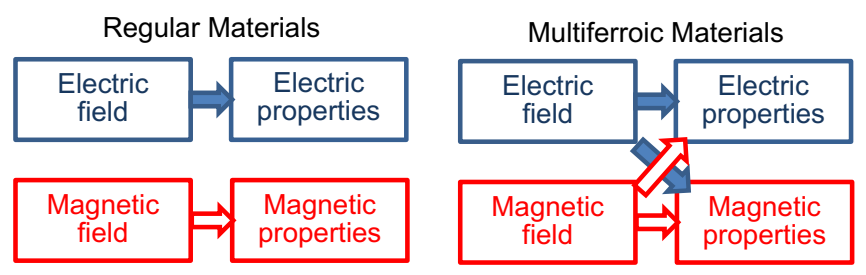

Figure 10. Uncoupled magnetic and electric properties of the ferroelectric and ferromagnetic materials are depicted to the left and the coupled magnetic and electric properties of magnetoelectric materials are depicted to the right [26].

As can be realized by controlling $V_{\mathrm{DC}}$ across the piezoelectric layer, we can achieve fast and effective tuning method to realize beam-steering. The magnetic and dielectric properties of the two ceramic phases are basically modified, changing the phase velocity and causing steering. That is, the magnetic properties of the ferrites can be tuned by an electric field applied to the piezoelectric phase [26]. This concept is depicted below in Figure 10.

To achieve good scanning, it is important to have good ME coupling coefficients. Indeed, large ME coupling coefficients have been reported for several $100 \mu \mathrm{m}$ thick YIG and PZT composites [19, 25, 26]. However, the fabrication of these $\mu \mathrm{m}$ thin films is very challenging and corresponding ME coefficients $(\mathrm{Oe} \mathrm{cm} / \mathrm{kV})$ depend on the interfacial bonding quality, sample thicknesses and strength of the applied voltage. Specifically, incorporation of given properties in the LWA design requires an experimental characterization of the ME coefficients for the manufactured substrates along a proper choice of the frequency for optimal antenna operation. A performance measure for these bi-layered composite is the ME coupling coefficient (in units of Oe $\mathrm{cm} / \mathrm{kV}$ ). This coefficient is a measure of the internal magnetic field change within the ferrite layer per unit electric field applied over the piezo electric layer. Higher coupling is preferred to attain wide angle scanning with minimal DC voltage control.

There are several magnetoelectric composites reported in the literature with good ME coupling coefficients [25-29]. Among them, the highest coupling was reported for $\mathrm{Ni}_{2} \mathrm{MnGa} / \mathrm{PMN}-\mathrm{PT}$ composites [29]. However, $\mathrm{Ni}_{2} \mathrm{MnGa}$ is metallic and therefore cannot be used as a substrate for the leaky-wave antenna due to its large ohmic losses (eddy 
currents). The second highest magnetoelectric coupling was reported for YIG/PMN-PT composites with a $\sim 100 \mu \mathrm{m}$ layer thicknesses. The fabrication of these micrometer $(\mu \mathrm{m})$ thin films is challenging and the corresponding $\mathrm{ME}$ coefficients $(\mathrm{Oe} \mathrm{cm} / \mathrm{kV}$ ) depend on the interfacial bonding quality, sample thicknesses and strength of the applied voltage. Yet, this heterostructure holds promise for microwave applications due to the YIG's low magnetic and dielectric losses.

We have already designed several leaky-wave antennas with wide angle scanning on low-loss ferrite substrates. A key challenge in realizing beam-steering was to adjust the magnetic properties of the ferrite substrates such that fast-waves can be supported by the microstrip line and, thus, radiate efficiently. However, the ferrite substrate was several mm thick. Accordingly, the realization of coupling parameters in required a $V_{\mathrm{DC}}$ on the order of $\mathrm{kV}$, viz. too large. On the other hand, using thinner substrates results in guided-waves that are strongly bound to the surface at low frequencies, implying poor antenna radiation. To use thinner samples and still obtain good radiation properties, we must operate at higher frequencies. To this end, we examined higher frequency usages for this LWA. Operating in the $X$-band is preferred as it covers the satellite uplink frequency band from 7.9 to $8.4 \mathrm{GHz}$ and the downlink band from 7.25 to $7.75 \mathrm{GHz}$. Higher operation frequencies will allow for even thinner ferrite and piezo-electric layers (several $100 \mu \mathrm{m}$ thick) and will operate with smaller bias voltages $(0-50 \mathrm{~V})$. Additionally, higher operation frequencies would allow for more unit-cells in the LWA design. Therefore, it would lead to increased directivity and gain.

It is encouraging that the growth of $100 \mu \mathrm{m}$ thick YIG samples have been reported in the literature using liquid phase epitaxial growth and we will be exploring this option for future [27]. In this method, thin YIG films are grown on another crystal sample (i.e. Gadolinium Gallium Garnet, GGG) having same crystal orientation. Then, the GGG layer is polished off to obtain the stand-alone YIG. Unfortunately, Ohio State University does not have the facility for liquid phase epitaxy. For the time being, our efforts are focused on designing LWAs supported on readily available YIG substrates. The thinnest YIG layer to be purchased from TCI ceramics is $0.38 \mathrm{~mm}$ thick and $5 \mathrm{~cm}$ long. In the next section, we demonstrate an initial leaky-wave antenna design on a single layer $2.34 \mathrm{~mm} \times$ $127 \mathrm{~mm} \times 0.38 \mathrm{~mm}\left(0.092^{\prime \prime} \times 5^{\prime \prime} \times 0.015^{\prime \prime}\right) \quad$ YIG substrate. This new design operates at $X$-band and can scan down to $65^{\circ}$ away from normal.

\subsection{Leaky-wave Antenna Operating at X-band}

As mentioned above, we designed a new LWA based on a single CRLH transmission line, operating at $X$-band. In our previous efforts, the LWA was always operated below ferromagnetic resonance (FMR) of the ferrite layer to utilize ferrite's permeability $\left(\mu_{r}>1\right.$ for $\left.f>f_{\mathrm{FMR}}\right)$ is larger and can be used for additional miniaturization. Miniaturization was necessary to attain good antenna performance at $L$-band with an electrically short layout (only $0.75 \lambda_{0}$ long). As mentioned before, the FMR is related to the external bias field $H_{0}$, saturation magnetization $4 \pi M_{s}$ of the ferrite and demagnetizing fields.

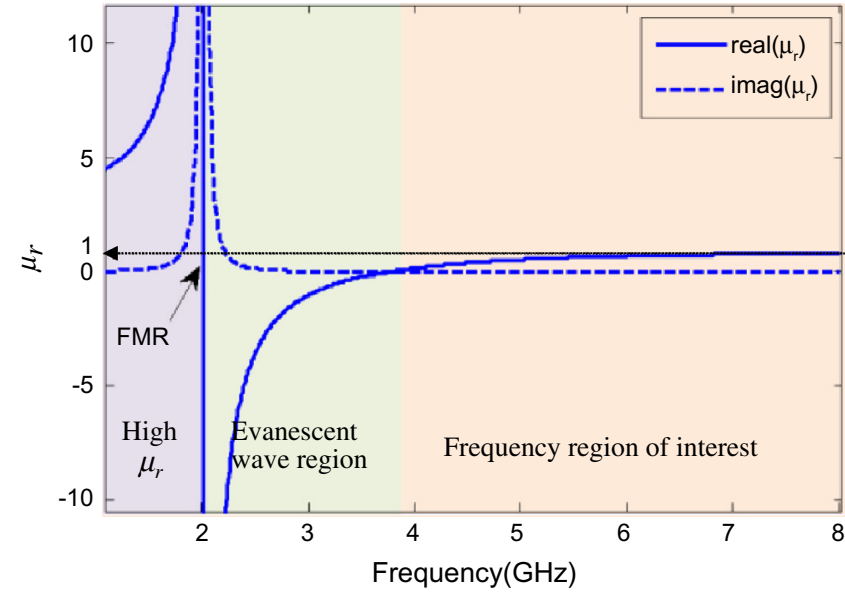

Figure 11. Relative permeability, $\mu_{r}$ of YIG substrate biased with $H_{\text {ext }}=2500$ Oe along its smallest dimension. Above $3.7 \mathrm{GHz}, \mu_{r}$ becomes positive again and allows for tunable operation at $X$-band controlled by the internal bias field $H_{0}$.

As an example, for ferrites biased normal to the ground plane, a practical external bias field of $H_{\text {ext }}=2500$ Oe would lead to an FMR frequency lower than $7 \mathrm{GHz}$ $\left(=\gamma H_{\text {ext }}, \gamma=2.8 \mathrm{MHz} / \mathrm{Oe}\right)$. Depending on the $4 \pi M_{s}$ of the ferrite substrate (i.e. $1780 \mathrm{G}$ for $\mathrm{YIG}$ ), the FMR frequency can be as low as $2 \mathrm{GHz}$, limiting the operation frequency of our previous LWAs. For $X$-band operation, we must have efficient radiation above FMR. Figure 11 depicts the permeability of YIG having $4 \pi M_{s}=1780 \mathrm{G}, \Delta H=20$ Oe biased with $H_{\text {ext }}=$ 2500 Oe along its smallest dimension $\left(H_{0}=H_{\text {ext }}-4 \pi M_{s}\right)$. We observe that above FMR $\left(\gamma H_{0} \approx 2 \mathrm{GHz}\right.$, $\left.H_{0}=H_{\text {ext }}-4 \pi M s\right)$, ferrite's relative permeability $\mu_{r}<0$ and would not support wave propagation (evanescent waves). However, when the operating frequency is larger than $\gamma \sqrt{H_{0}\left(H_{0}+4 \pi M_{s}\right)} \approx 3.7 \mathrm{GHz}$, the permeability becomes positive again $\left(0<\mu_{r}<1\right)$, allowing operation at the $C$ and $X$-band (4-8 GHz). Of course, the ferrite's permeability is still controlled by the bias field and this property is essential for frequency independent beamscanning.

By operating at $X$-band, we designed a new LWA layout using a single CRLH transmission line. This new design is comprised of an ultra-thin polycrystal YIG substrate $\left(\varepsilon_{r}=15\right.$, $\left.\tan \delta=10^{-4}, 4 \pi M_{s}=1780 \mathrm{G}, \Delta H=20 \mathrm{Oe}\right) 0.38 \mathrm{~mm}(15 \mathrm{mil})$ thick placed on 5 mil thick RT-Duroid 3010 superstrate $\left(\varepsilon_{r}=10.2, \tan \delta=0.0022\right)$. The CRLH TL is printed on both sides of the superstrate as indicated in Figure 12.

Similar to our previous design, the overlapping transmission line sections are employed to realize series capacitance $\left(C_{\mathrm{se}}\right)$ creating a series resonance with the inherent inductance of the transmission line. Shunt stubs are also used to create a second resonance with the capacitance of the transmission lines. When these two resonances overlap, a continuous beam-scanning is attained from the backward endfire direction to forward endfire direction.

Additionally, due to the left handed nature of this artificial transmission line, a high-pass filter is realized with 

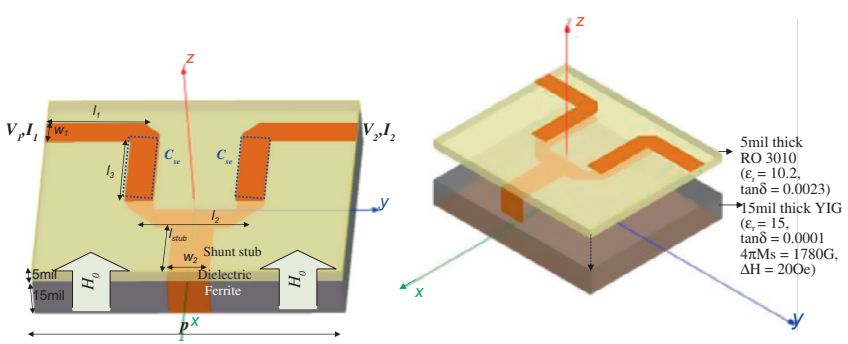

Figure 12. Unit-cell of the proposed coupled CRLH for operation at $X$-band, the geometry is comprised of a 5 mil thick PCB (RO 3010) placed on a 15 mil thick YIG biased normal to the ground plane. Its exploded view explicitly shows the constituent layers. The dimensions are: $l_{1}=32.5, l_{2}=40, w_{1}=10, w_{2}=15, l_{3}=37, l_{\text {stub }}=$ 30 (mils).

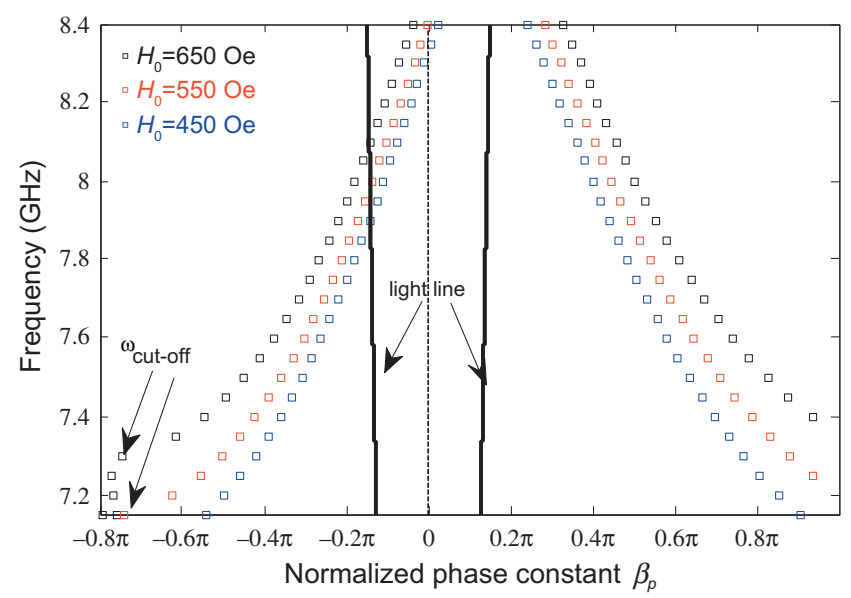

Figure 13. Dispersion curves of the unit-cell design in Figure 12 calculated for 3 different bias field strengths, $H_{0}=450,550$, 650 Oe. Tunability of the dispersion curves is limited because the operation frequency is far away from the ferromagnetic resonance of the YIG substrate.

$\omega_{\text {cutoff }}=\frac{1}{\sqrt{C_{\mathrm{se}} L_{\text {shunt }}}}$, where $C_{\mathrm{se}}$ is dependent on overlapping TL arms and $L_{\text {shunt }}$ is associated with the shunt stub of length $l_{\text {stub. }}$.

Figure 13 depicts the dispersion curves of the proposed unit-cell calculated at three different bias field values $H_{0}=450,550,650$ Oe using full-wave simulations. As would be expected, the sensitivity of the dispersion curves to the bias field $H_{0}$ is limited since the operating frequency is very far away from the FMR frequency of the YIG. Therefore, the low-end cut-off frequency is chosen close to the operation frequency to tune the dispersion lines by changing $\omega_{\text {cutoff }}$. This new LWA design has a unit-cell length $(p)$ of only 105 mil $\left(\lambda_{0} / 14\right)$ and is only 20 mil $\left(\lambda_{0} / 75\right)$ thick.

The realized LWA is depicted in Figure 14a, and consists of 48 unit-cells cascaded in the direction of wave propagation (along $y$ axis), corresponding to an overall length of 5.04" ( $3.45 \lambda_{0}$ at $8.1 \mathrm{GHz}$ ). The length is specifically chosen to be shorter than our $6^{\prime \prime}$ long magnet so that the available is fully when it is fabricated and tested. The performance of this ferrite CRLH was calculated using HFSS v14 and simulated results show that this new design provides $65^{\circ}$ scanning at $8.1 \mathrm{GHz}$

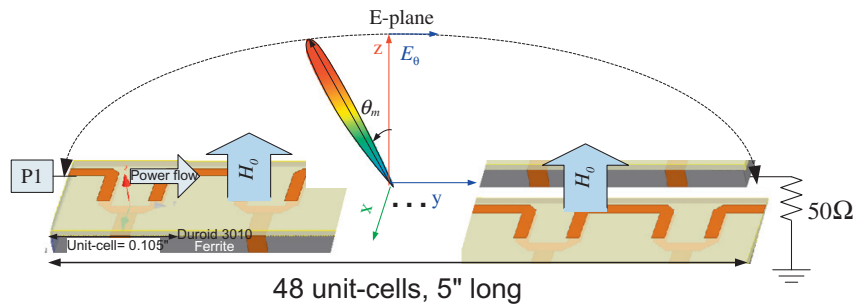

(a)

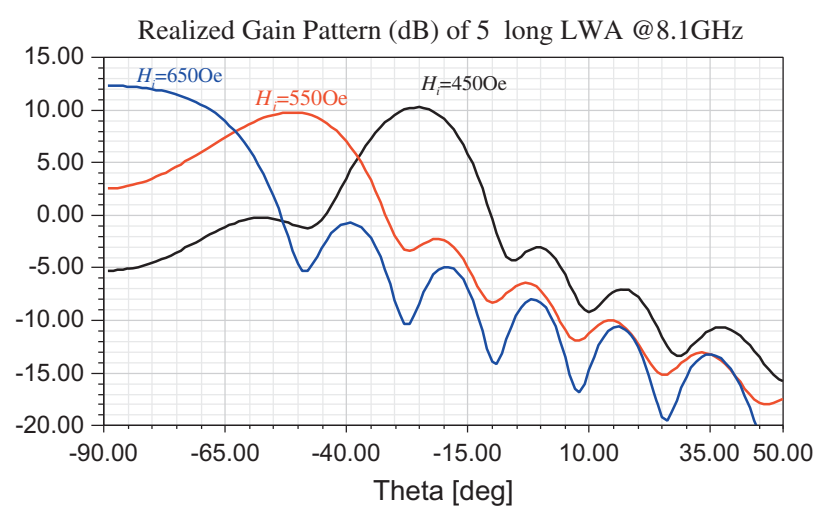

(b)

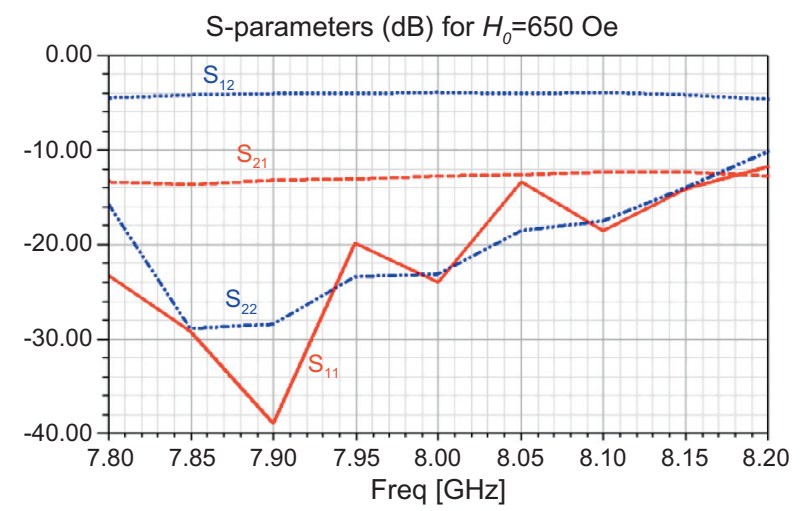

(c)

Figure 14. (a) Visualization of the LWA comprised of 48 unit-cells, fed at port 1 and terminated with $50 \Omega$ at the unused port. (b) Beamscanning performance of the proposed LWA at $8.1 \mathrm{GHz}$ by changing the bias field $\pm 100 \mathrm{Oe}$. (c) $S$-parameters for the given ferrite CRLH layout between $7.8 \mathrm{GHz}$ and $8.2 \mathrm{GHz}$. note that $S_{11}$ is less than $-10 \mathrm{~dB}$ over the bandwidth.

with only \pm 100 Oe variation of the bias field as depicted in Figure 14b. Similar scanning can be attained over the Satcom uplink band $\left(7.9 \mathrm{GHz}<f_{\text {operation }}<8.4 \mathrm{GHz}\right)$ by properly tuning the bias field. Unfortunately, scanning cannot be realized for the 7.25-7.75 GHz downlink band using this design. For operation within this frequency range, we require the bias field that is much smaller. In that case, the ferrite might exhibit losses due to partial saturation. Therefore, it is more reasonable to have another CRLH TL with optimized performance at downlink frequencies.

The $S$-parameters of the proposed LWA were also simulated and are shown in Figure 14c. As seen, $S_{11}$ is less than $-10 \mathrm{~dB}$ between $7.8 \mathrm{GHz}$ and $8.2 \mathrm{GHz}$ and $S_{21}$ is around $-14 \mathrm{~dB}$. 


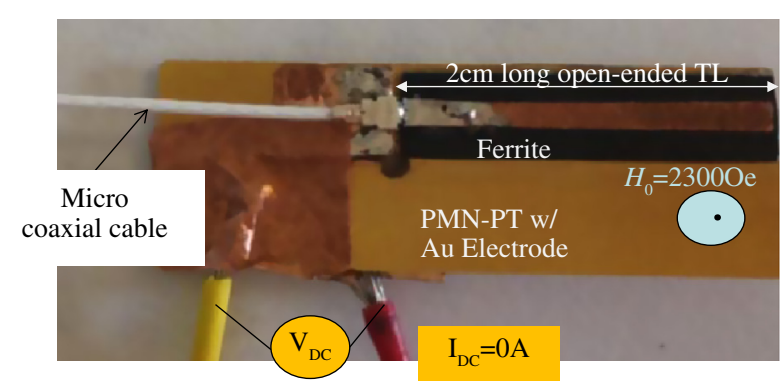

(a)

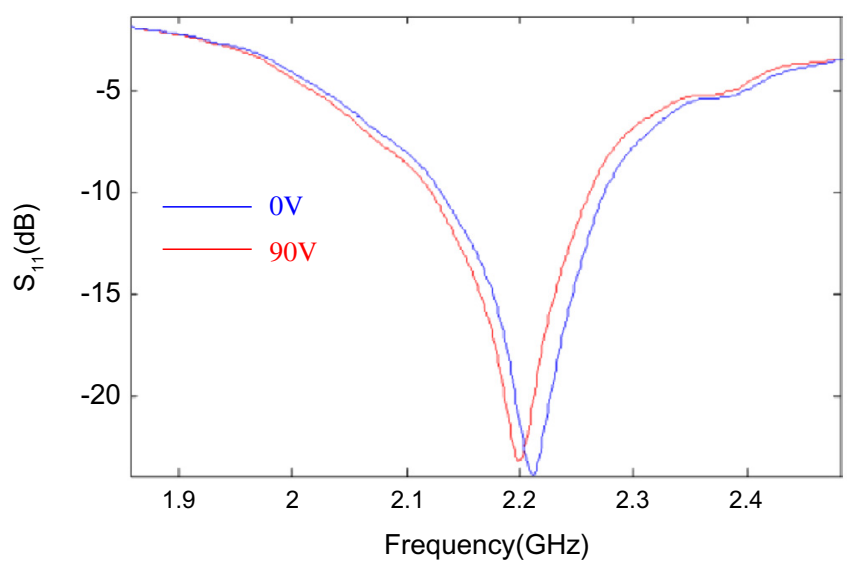

(b)

Figure 15. (a) In house manufactured open ended TL resonator based on magnetoelectric composite comprised of 15 mil thick YIG $\left(\varepsilon_{r}=15, \tan \delta=1 \mathrm{e}-4,4 \pi M_{s}=1780 \mathrm{G}, \Delta H=20 \mathrm{Oe}\right)$ film bonded to 15 mil thick PMN-PT layer. (b) Corresponding input reflection coefficients for $V_{\mathrm{DC}}=0$ and $90 \mathrm{~V}$.

Radiation efficiency is quite good. Specifically, it is more than $75 \%$ over the operation bandwidth.

We remark that the used ferrite substrate used is four times thicker $(380 \mu \mathrm{m})$ than the epitaxially grown $(100 \mu \mathrm{m})$ films reported in the literature. Yet it was the thinnest sample commercially available to us. The $V_{\mathrm{DC}}$ required for a change of \pm 50 Oe in magnetic field strength needs to be experimentally determined since there is no reported data in the literature on thick YIG samples. A simple characterization method is presented next.

\subsection{Magneto-electric coupling coefficient characterization}

The strength of the ME effect depends on the crystal structure of the two phases, interfacial bonding quality, thickness of the layers, orientation of the dc magnetic field (i.e. in plane or normal), and the strength of the DC voltage bias. Optimization of the ME coupling would require experimenting all given parameters. Among these, interfacial bonding quality is the most important parameter in the overall performance since coupling is due to the mechanical interaction through surface tension. This might necessitate epitaxial growth of ferrite layer on the piezo layer to increase the bonding quality. For initial testing, these materials can be supplied from material companies. However, the thickness of these commercially available samples is several hundred microns. This would increase the DC voltage needed to attain required FMR shift in ferrite substrate. Characterization of these heterostructures can be easily performed via simple $S$-parameters measurements.

For experimental validation, we ordered off-the-shelf YIG (of 15 mil thickness) and PMN-PT films (of 15 mil thickness) from TCI ceramics and HC Materials. PMN-PT layer has Au coating on top and bottom surfaces to apply the DC voltage bias. These two layers were bonded using epoxy and a $2 \mathrm{~cm}$ long an open ended microstrip line was placed on the ferrite substrate. DC magnetic field was applied normal to the substrate using permanent magnet. Subsequently, input reflection coefficient was measured as the $\mathrm{V}_{\mathrm{DC}}$ was changed. Figure depicts the final magnetoelectric composite layout and corresponding reflection coefficient $S_{11}$ for $V_{\mathrm{DC}}=0$ and $90 \mathrm{~V}$. as observed, tunability is limited and the shift in $S_{11}$ corresponds to 5 Oe change in internal magnetic field $H_{i}$. This is less than what is reported in [28], where 44 Oe change in $H_{i}$ is reported. The discrepancy is attributed to the thickness of the ordered YIG layer (four times thicker) and polycrystalline nature of it.

To attain larger magnetoelectric couplings, it is necessary to have very thin single crystal YIG films. The incorporation of these materials in antenna designs will be game-changing and lead to multi-functional, low-profile and cost efficient novel antennas. Yet, challenges exist. Among them are realizing optimal antenna performances to have efficient, wide-band and directive radiation for the aforementioned applications (i.e. radars, high data rate communications) and achieving wide angle beam steering using only small variation in the applied DC voltage.

\section{Conclusion}

In this paper, we proposed a new class of miniaturized nonreciprocal LWAs comprised of coupled TLs on a ferrite substrate to achieve wide angle beamscanning capability. One of our designs was a regular periodic LWA that radiates using the fast higher order space harmonic of the dominant slow wave mode. A key issue limiting the performance of this ferrite LWA was the non-uniformities in the external and internal magnetic fields. To alleviate this issue, we proposed a novel miniaturized coupled CRLH LWA on a ferrite substrate with wide angle beamscanning. Scanning using the ferrite CRLH LWA was achieved by slightly varying the magnetic bias field. We achieved wide angle scanning with minimal bias field control by increasing the sensitivity of the leaky-waves' phase constant to vary the bias field. Additionally, coupling between the TL pair and the nonreciprocity of the ferrite led to concurrent reception and transmission at different ports. The TX and RX antenna patterns were successfully scanned over $80^{\circ}$ in the $E$-plane by changing the bias field strength only $\pm 50 \mathrm{Oe}$. This was done by mechanically changing the distance between the LWA and the magnet providing the external bias.

A new class of magnetoelectrics-based leaky-wave antennas (LWA) were also proposed for wide angle beam-steering 
via electrical tuning. Electrical tuning is fast, reliable and of low power. As mentioned before, challenges still exist in operating with low DC voltages and in broadening the scanning range while keeping magnetic losses to minimum. We believe that the incorporation of these materials in antenna designs will lead to multi-functional, low-profile and cost efficient novel antennas.

Acknowledgements. This work was supported by CERDEC Space \& Terrestrial Communications Directorate, Aberdeen Proving Grounds, MD and the National Science Foundation under Grant \#IIP1134641

\section{References}

1. IEEE Transactions on Antennas and Propagation, Special Issue on Metamaterials, 51 (2003) 10

2. D. Polder, Phil. Mag. 40 (1949) 99-115.

3. H. Gundel, H. Riege, E.J.N. Wilson, J. Handerek, K. Zioutas, Nucl. Instrum. Methods Phys. Res. A 280 (1989) 1-6.

4. X. Zuo, H. How, S.A. Oliver, C. Vittoria, IEEE Trans. Magn. 37 (2001) 4.

5. J.L. Volakis, C.C. Chen, K. Fujimoto, Small antennas: Miniaturization techniques and applications, McGraw-Hill, 2010.

6. R.C. Hansen, in Antenna Engineering Handbook, edited by J.L. Volakis, 4th ed. McGraw-Hill, New York, 2007.

7. A.A. Oliner, David R. Jackson, in Antenna Engineering Handbook, edited by J.L. Volakis, 4th ed., McGraw-Hill, New York, 2007.

8. S. Lim, C. Caloz, Tatsuo Itoh, IEEE Trans. Microw. Theory Tech. 52 (2004) 12.

9. B.C.C. Chang, Y. Qian, T. Itoh, in Proceedings of the IEEE Int. Symp. Antennas Propagation Society, Orlando, FL, vol. 4, 1999, pp. 2694-2697.

10. D. Sievenpiper, J. Schaffner, J.J. Lee, S. Livingston, IEEE Antennas Wirel. Propag. Lett. 1 (2002)

11. N. Apaydin, L. Zhang, K. Sertel, J.L. Volakis, Proceedings of the IEEE Int. Symp. Antennas Propagation Society, Chicago, IL, 2012, pp. 1-4.

12. N. Apaydin, K. Sertel, J.L. Volakis, Nonreciprocal leaky-wave antenna based on coupled microstrip lines on a non-uniformly biased ferrite substrate, IEEE Trans. Antennas Propag. 61 (2013) 7 .

13. H. Maheri, M. Tsutsumi, N. Kumagai, Experimental studies of magnetically scannable leaky-wave antennas having a corrugated ferrite slab/dielectric layer structure, IEEE Trans. Antennas Propag. 36, 7 (1988) 911-917.

14. T. Kodera, C. Caloz, Uniform ferrite-loaded open waveguide structure with CRLH response and its application to a Novel backfire-to-endfire leaky-wave antenna, IEEE Trans. Microwave Theory Tech. 57 (2009) 784-795.
15. P. Baccarelli, C. Di Nallo, F. Frezza, A. Galli, P. Lampariello, Attractive features of leaky-wave antennas based on ferriteloaded open waveguides, Antennas and Propagation Society Int. Symp., Montreal, QC, 1997, pp. 1442-1445.

16. T. Kodera, C. Caloz, Integrated leaky-wave antenna-duplexer/ diplexer using crlh uniform ferrite-loaded open waveguide, IEEE Trans. Antennas Propag. 58, 8 (2010) 2508-2514.

17. T. Ueda, K. Horikawa, M. Akiyama, M. Tsutsumi, Nonreciprocal phase-shift composite right/left handed transmission lines and their application to leaky wave antennas, IEEE Trans. Antennas Propag. 57, 7 (2009) 1995-2005.

18. C. Caloz, T. Itoh, "Radiated-wave applications" in Electromagnetic metamaterials: transmission line theory and microwave applications, Wiley, Hoboken, NJ, 2006, pp. 261-310.

19. C. Nan, M.I. Bichurin, S. Dong, D. Viehland, G. Srinivasan, Multiferroic magnetoelectric composites: Historical perspective, status, and future directions, J. Appl. Phys. 103, 031101 (2008) $1-32$.

20. S. Paulotto, P. Baccarelli, F. Frezza, D.R. Jackson, A novel technique for open-stopband suppression in 1-d periodic printed leaky-wave antennas, IEEE Trans. Antennas Propag. 57, 7 (2009) 1894-1906.

21. N. Apaydin, L. Zhang, K. Sertel, J.L. Volakis, Experimental validation of frozen modes guided on printed coupled transmission lines, IEEE Trans. Microwave Theory Tech. 60, 6 (2012) 1513-1518.

22. T. Ueda, K. Horikawa, M. Akiyama, M. Tsutsumi, Nonreciprocal phase-shift composite right/left handed transmission lines and their application to leaky wave antennas, IEEE Trans. Antennas Propag. 577 (2009) 1995-2005.

23. R.I. Joseph, E. Schlomann, Demagnetizing field in nonellipsoidal bodies, J. Appl. Phys. 36, 5 (1965) 1579-1592.

24. N. Apaydin, K. Sertel, J.L. Volakis, Nonreciprocal and magnetically scanned leaky-wave antenna using coupled CRLH lines, IEEE Trans. Antennas Propag. 62 (2014) 6.

25. Y.K. Fetisov, G. Srinivasanan, Electric field tuning characteristics of a ferrite-piezoelectric microwave resonator, Appl. Phys. Lett. 88 (2006) 143503.

26. G. Srinivasan, Electric field tunable microwave and $\mathrm{mm}$ wave ferrite devices, Final Report on ONR Contract No. N00014-061-0167, Oakland University, 2010.

27. V.G. Harris, A. Geiler, Y. Chen, S.D. Yoon, M. Wu, A. Yang, Z. Chen, P. He, P.V. Parimi, X. Zuo, C.E. Patton, M. Abe, O. Acher, C. Vittoria, Recent advances in processing and application of microwave ferrites, Elsevier J. Magnetism Magn. Mater. 321 (2009) 2035-2047.

28. A.S. Tatarenko, V. Gheevarughese, G. Srinivasanan, Magnetoelectric microwave bandpass filter, Electron. Lett. 42 (2006) 9.

29. Y. Chen, J. Wang, M. Liu, N.X. Sun, C. Vittoria, V.G. Harris, Giant magnetoelectric coupling and e-field tunability in a laminated Ni2mnga/lead-magnesium-niobate-lead titanate multiferroic heterostructure, Appl. Phys. Lett. 93, 112502 (2008) $1-4$.

Cite this article as: Apaydin N, Sertel K \& Volakis JL: Nonreciprocal and magnetically scanned miniaturized leaky-wave antennas using coupled transmission lines. EPJ Appl. Metamat. 2014, 1, 3. 